07

\title{
Увеличение фототока Ga(In)As-субэлемента в многопереходных солнечных элементах GalnP/Ga(In)As/Ge
}

\author{
(C) С.А. Минтаиров, В.М. Емельянов, Н.А. Калюжный, М.З. Шварц, В.М. Андреев \\ Физико-технический институт им. А.Ф. Иоффре РАН, Санкт-Петербург, Россия \\ E-mail: mintairov@scell.ioffe.ru
}

Поступило в Редакцию 22 июля 2019г.

В окончательной редакции 2 сентября 2019г.

Принято к публикации 18 сентября 2019г.

\begin{abstract}
Проведено экспериментальное и теоретическое исследование спектральных характеристик $\mathrm{Ga}(\mathrm{In}) \mathrm{As}$-субэлемента трехпереходных солнечных элементов $\mathrm{GaInP} / \mathrm{Ga}(\mathrm{In}) \mathrm{As} / \mathrm{Ge}$. Показано, что использование слоя широкозонного „окна“ с оптимизированной толщиной $\left(\mathrm{Ga}_{0.51} \mathrm{In}_{0.49} \mathrm{P}-100 \mathrm{~nm}, \mathrm{Al}_{0.4} \mathrm{Ga}_{0.6} \mathrm{As}-110 \mathrm{~nm}\right.$, $\mathrm{Al}_{0.8} \mathrm{Ga}_{0.2} \mathrm{As}-115 \mathrm{~nm}$ ) для $\mathrm{Ga}(\mathrm{In})$ Аs-субэлемента позволяет увеличить его фототок на величину порядка $0.5 \mathrm{~mA} / \mathrm{cm}^{2}$, замена материала слоя тыльного потенциального барьера GaInP-субэлемента с $\mathrm{Al}_{0.53} \operatorname{In}_{0.47} \mathrm{P}$ на $p^{+}-\mathrm{Ga}_{0.51} \mathrm{In}_{0.49} \mathrm{P}$ или $\mathrm{AlGaAs}$ позволяет повысить ток короткого замыкания $\mathrm{Ga}(\mathrm{In}) \mathrm{As}$-субэлемента еще на величину порядка $0.8 \mathrm{~mA} / \mathrm{cm}^{2}$, а использование в туннельном диоде широкозонного слоя $n^{++}-\mathrm{Ga}_{0.51} \mathrm{In}_{0.49} \mathrm{P}$ вместо $n^{++}$-GaAs повышает фототок на величину порядка $1 \mathrm{~mA} / \mathrm{cm}^{2}$.
\end{abstract}

Ключевые слова: солнечный элемент, математическое моделирование, фототок, субэлемент, арсенид галлия.

DOI: 10.21883/PJTF.2019.24.48802.17996

Сегодня среди фотопреобразователей солнечной энергии наибольшим КПД в промышленном производстве обладают многопереходные (МП) солнечные элементы (CЭ) $\mathrm{GaInP} / \mathrm{Ga}(\mathrm{In}) \mathrm{As} / \mathrm{Ge}$ с тремя фотоактивными $p-n$-переходами, выполненными на основе согласованных по параметру решетки материалов $\mathrm{Ga}_{0.51} \mathrm{In}_{0.49} \mathrm{P}$, $\mathrm{Ga}_{0.99} \mathrm{In}_{0.01} \mathrm{As}$ и $\mathrm{Ge}$ и соединяющих их встречно включенных $n^{++}-p^{++}$-туннельных диодов (ТД) [1-7]. При этом лабораторный рекорд эффективности $\sim 46 \%$ (AM1.5D) был достигнут для четырехпереходного СЭ [8].

Основным фактором, ограничивающим эффективность преобразования МП СЭ на основе $\mathrm{GaInP} / \mathrm{Ga}(\mathrm{In}) \mathrm{As} / \mathrm{Ge}$, является низкий или недостаточный ток короткого замыкания среднего субэлемента, выполненного на основе $\mathrm{Ga}_{0.99} \mathrm{In}_{0.01} \mathrm{As}$ (далее $\mathrm{Ga}(\mathrm{In}) \mathrm{As}$ ). Очевидно, что его увеличение будет оказывать значительное влияние как на КПД многопереходного СЭ, так и на его радиационную стойкость [9].

Уменьшение количества фотонов, приходящих в средний субэлемент структуры $\mathrm{GaInP} / \mathrm{Ga}(\mathrm{In}) \mathrm{As} / \mathrm{Ge}$, связано в первую очередь с поглощением света в слоях „верхнего“ туннельного диода и его отражением от гетерограниц слоев, находящихся между фотоактивными $p-n$-переходами, выполненными из материалов $\mathrm{Ga}_{0.51} \mathrm{In}_{0.49} \mathrm{P}$ и $\mathrm{Ga}(\mathrm{In}) \mathrm{As}$. Уменьшение отражения от гетерограницы возможно за счет использования эпитаксиального слоя с показателем преломления, значение которого находится между значениями показателей преломления материалов, образующих просветляемую границу. Если это условие не выполняется, то такой слой будет увеличивать амплитуду отраженной волны, играя роль селективного отражателя.
Настоящая работа посвящена проблеме минимизации оптических потерь на гетерогранице объемных слоев GaInP и $\mathrm{Ga}(\mathrm{In}) \mathrm{As}$ в структуре МП СЭ $\mathrm{GaInP} / \mathrm{Ga}(\mathrm{In}) \mathrm{As} / \mathrm{Ge}$ для повышения фотогенерированного тока среднего субэлемента $\mathrm{Ga}(\mathrm{In}) \mathrm{As}$.

Структуры МП СЭ выращивались методом газофазной эпитаксии из металлоорганических соединений. Дизайн структур и чипов СЭ, а также подробности технологии эпитаксиального роста представлены в [10,11]. В настоящей работе варьировался дизайн гетероструктуры среднего субэлемента МП СЭ и расположенных над ним функциональных слоев, а именно исследовались различные варианты для широкозонного „окна“ $\left(\mathrm{Ga}_{0.51} \mathrm{In}_{0.49} \mathrm{P}, \mathrm{Al}_{0.4} \mathrm{Ga}_{0.6} \mathrm{As}, \mathrm{Al}_{0.8} \mathrm{Ga}_{0.2} \mathrm{As}\right)$ среднего субэлемента, составов широкозонных слоев верхнего туннельного диода $\left(\mathrm{Ga}_{0.51} \mathrm{In}_{0.49} \mathrm{P}\right.$ и $\left.\mathrm{GaAs}\right)$, расположенного над широкозонным окном среднего субэлемента $\mathrm{Ga}(\mathrm{In}) \mathrm{As}$, а также варианты тыльного потенциального барьера $\left(\mathrm{Al}_{0.53} \mathrm{In}_{0.47} \mathrm{P}, \mathrm{Ga}_{0.51} \mathrm{In}_{0.49} \mathrm{P}, \mathrm{AlGaAs}\right)$ верхнего субэлемента. Количественно эффективность проводимых оптимизаций оценивалась по результатам измерений спектральных зависимостей внешнего квантового выхода фотоответа $\mathrm{Ga}(\mathrm{In}) \mathrm{As}$-субэлементов в составе МП СЭ и рассчитываемым значениям фототока для солнечного спектра АМ0.

Для определения количества проходящих и поглощенных в субэлементах фотонов картина электромагнитного поля в структуре МП СЭ формировалась с помощью метода матриц Абелеса [12]. Расчеты спектров фотоответа проводились с использованием теории фотовольтаического эффекта [13]. Параметры расчетов, описание методики моделирования структур МП СЭ представлены в [14]. 


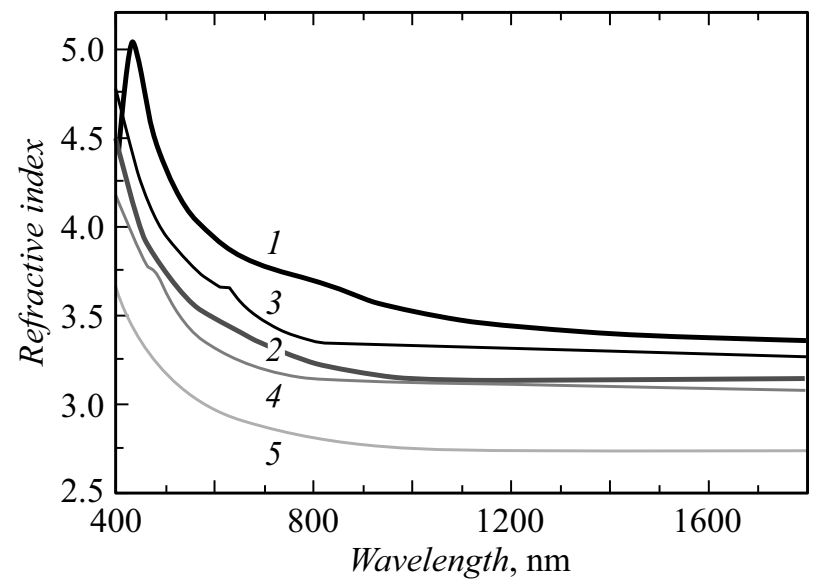

Рис. 1. Дисперсионные зависимости показателей преломления слоев, используемых в гетероструктурах многопереходных солнечных элементов. $1-\mathrm{GaAs}\left(\mathrm{Ga}_{0.99} \mathrm{In}_{0.01} \mathrm{As}\right)$, $2-\mathrm{Ga}_{0.51} \mathrm{In}_{0.49} \mathrm{P}, 3-\mathrm{Al}_{0.4} \mathrm{Ga}_{0.6} \mathrm{As}, 4-\mathrm{Al}_{0.8} \mathrm{Ga}_{0.2} \mathrm{As}$, $5-\mathrm{Al}_{0.53} \operatorname{In}_{0.47} \mathrm{P}$.

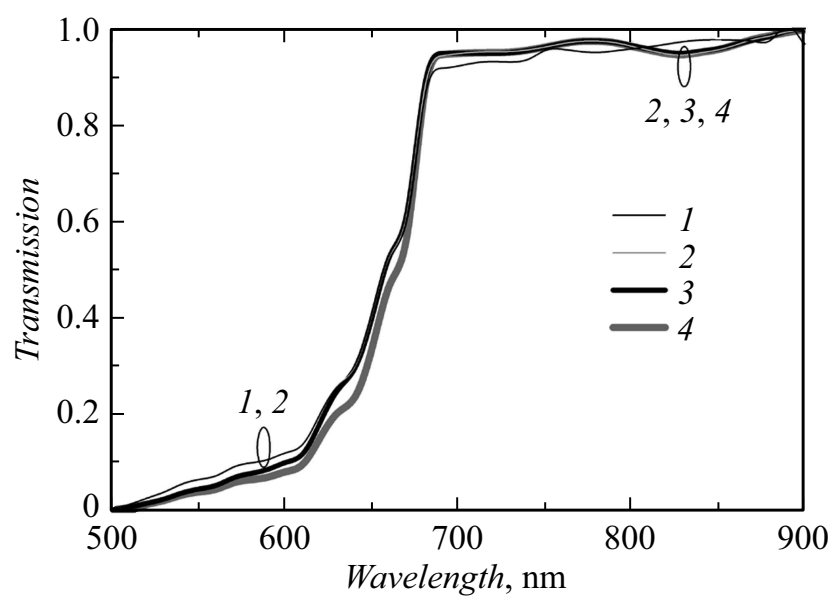

Рис. 2. Расчетные спектральные зависимости коэффициента

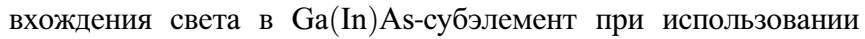
слоя широкозонного окна $\mathrm{Al}_{0.8} \mathrm{Ga}_{0.2} \mathrm{As}$ толщиной $30 \mathrm{~nm}$ (1), a также оптимизированных по толщине слоев $\mathrm{Al}_{0.8} \mathrm{Ga}_{0.2} \mathrm{As}$ толщиной $115 \mathrm{~nm}(2), \mathrm{Al}_{0.4} \mathrm{Ga}_{0.6} \mathrm{As}$ толщиной $110 \mathrm{~nm}$ (3) и $\mathrm{Ga}_{0.51} \mathrm{In}_{0.49}$ Р толщиной $100 \mathrm{~nm}(4)$.

Наличие между субэлементами различных функциональных слоев позволяет уменьшить оптические потери в МП СЭ за счет варьирования их толщины и просветления гетерограниц. Однако, как правило, слой тыльного потенциального барьера верхнего субэлемента, слои туннельного диода и слой широкозонного окна среднего субэлемента СЭ не просветляют границу объемных материалов $\mathrm{GaInP}$ и $\mathrm{Ga}(\mathrm{In}) \mathrm{As}$.

Дисперсионные зависимости показателей преломления материалов МП СЭ (рис. 1) позволяют сделать вывод, что граница GaInP-Ga(In)As может быть просветлена слоем $\mathrm{Al}_{0.4} \mathrm{Ga}_{0.6}$ As. Именно этот материал используется в качестве $p$-слоя туннельного диода, обеспе- чивающего низкоомную развязку субэлементов МП СЭ. При этом толщина слоев ТД не превышает $15-20 \mathrm{~nm}$, что минимизирует поглощение в них полезного излучения. Для эффективной работы слоя широкозонного окна среднего субэлемента его толщина должна быть $30 \mathrm{~nm}$ или более. Таким образом, использование $\mathrm{Al}_{0.4} \mathrm{Ga}_{0.6} \mathrm{As}$ в качестве широкозонного окна может уменьшить внутреннее отражение и просветлить гетерограницу $\mathrm{GaInP}-\mathrm{Ga}(\mathrm{In}) \mathrm{As}$.

Однако проведенные расчеты показали, что для просветления этой гетерограницы толщина слоя широкозонного окна из $\mathrm{Al}_{0.4} \mathrm{Ga}_{0.6} \mathrm{As}$ и других материалов должна быть порядка $100 \mathrm{~nm}$ (рис. 2). Использование слоя $\mathrm{Al}_{0.4} \mathrm{Ga}_{0.6} \mathrm{As}$ такой толщины приведет к заметному поглощению полезного излучения в нем. В случае уменьшения толщины будут возрастать потери, связанные с отражением фотонов от гетерограницы $\mathrm{GaInP}-\mathrm{Ga}(\mathrm{In}) \mathrm{As}$. Этот эффект показан на примере более широкозонного слоя $\mathrm{Al}_{0.8} \mathrm{Ga}_{0.2} \mathrm{As}$ (рис. 2, кривые 1,2$)$, который поглощает меньшее количество фотонов. Использование оптимизированных толщин слоя широкозонного окна $\left(\mathrm{Ga}_{0.51} \mathrm{In}_{0.49} \mathrm{P}-100 \mathrm{~nm}\right.$, $\left.\mathrm{Al}_{0.4} \mathrm{Ga}_{0.6} \mathrm{As}-110 \mathrm{~nm}, \mathrm{Al}_{0.8} \mathrm{Ga}_{0.2} \mathrm{As}-115 \mathrm{~nm}\right)$ увеличивает пропускание в области от 675 до $900 \mathrm{~nm}$, что позволяет повысить ток короткого замыкания $\mathrm{Ga}(\mathrm{In}) \mathrm{As}-$ субэлемента на величину порядка $0.5 \mathrm{~mA} / \mathrm{cm}^{2}$ (AM0).

Однако использование узкозонных по отношению к $\mathrm{Al}_{0.8} \mathrm{Ga}_{0.2} \mathrm{As}$ материалов (GaInP или $\left.\mathrm{Al}_{0.4} \mathrm{Ga}_{0.6} \mathrm{As}\right)$ в качестве слоя широкозонного окна будет приводить к небольшому снижению количества проходящих в $\mathrm{Ga}(\mathrm{In})$ As-субэлемент фотонов в области $500-675 \mathrm{~nm}$ (рис. 2), что связано с их поглощением в этом слое. При этом лучшее структурное совершенство материала $\mathrm{GaInP,} \mathrm{связанное} \mathrm{с} \mathrm{отсутствием} \mathrm{алюминия,} \mathrm{позволяет}$ фотогенерированном в слое $\mathrm{GaInP}$ широкозонного окна

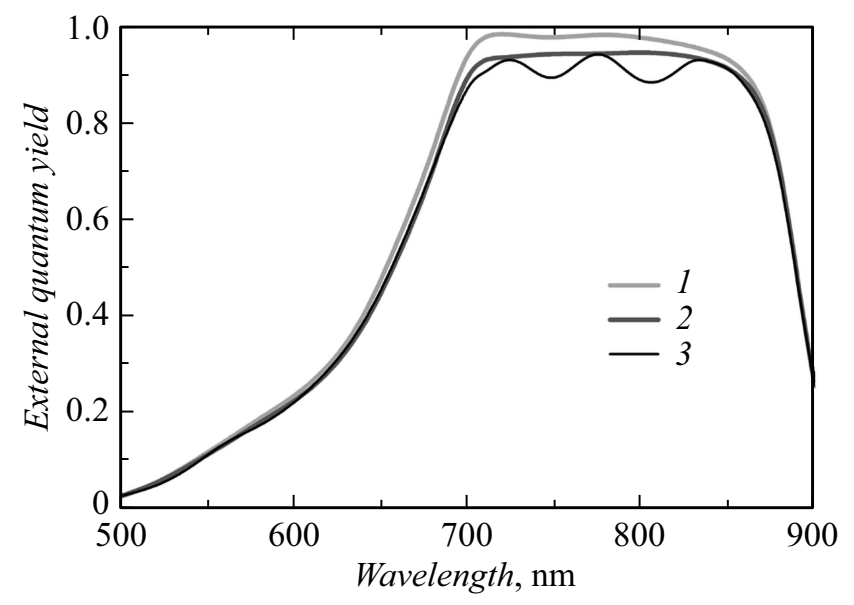

Рис. 3. Экспериментальные спектральные зависимости внешнего квантового выхода фотоответа $\mathrm{Ga}(\mathrm{In}) \mathrm{As}$-субэлемента МП СЭ $\mathrm{GaInP} / \mathrm{Ga}(\mathrm{In}) \mathrm{As} / \mathrm{Ge}$ в случае использования широкозонного окна $\mathrm{Ga}_{0.51} \mathrm{In}_{0.49} \mathrm{P}$ толщиной $100(1,2)$ и $30 \mathrm{~nm}$ (3), а также $n$-слоя верхнего туннельного диода $\mathrm{Ga}_{0.51} \mathrm{In}_{0.49} \mathrm{P}$ толщиной $15 \mathrm{~nm}(1)$ и GaAs толщиной $15 \mathrm{~nm}(2,3)$. 
носителям диффундировать до границы с фотоактивным $\mathrm{Ga}(\mathrm{In}) \mathrm{As} p-n$-переходом и дать вклад в фототок. Возможность собирания носителей, фотогенерированных в слое GaInP, делает этот материал наиболее оптимальным для широкозонного окна $\mathrm{Ga}(\mathrm{In}) \mathrm{As}$-субэлемента.

Поглощение полезного для $\mathrm{Ga}(\mathrm{In}) \mathrm{As-cyбэлемента} \mathrm{из-}$ лучения в слоях верхнего туннельного диода также снижает ток короткого замыкания этого субэлемента, поэтому для увеличения пропускания света верхним ТД возможна замена слоя $n^{++}$-GaAs на $n^{++}-\mathrm{Ga}_{0.51} \mathrm{In}_{0.49} \mathrm{P}$, который обладает большей шириной запрещенной зоны. Согласно проведенным расчетам, ток короткого замыкания $\mathrm{Ga}(\mathrm{In}) \mathrm{As}$-субэлемента повышается на величину порядка $1 \mathrm{~mA} / \mathrm{cm}^{2}$ (AM0) за счет использования такого слоя.

Заметное уменьшение количества достигающих $\mathrm{Ga}(\mathrm{In}) \mathrm{As}$-субэлемента фотонов происходит при использовании материала $\mathrm{Al}_{0.53} \operatorname{In}_{0.47} \mathrm{P}$ между GaInP- и $\mathrm{Ga}(\mathrm{In}) \mathrm{As}$-субэлементами. Это связано с тем, что показатель преломления AlInP ниже показателей преломления $\mathrm{GaInP}$ и $\mathrm{Ga}(\mathrm{In}) \mathrm{As}$ (рис. 1). Поэтому в МП СЭ в качестве тыльного потенциального барьера верхнего перехода целесообразно использовать слой $p^{+}$-GaInP или $\mathrm{AlGaAs}$. Это, согласно расчетам, позволяет повысить ток короткого замыкания $\mathrm{Ga}(\mathrm{In}) \mathrm{As}$-субэлемента еще на величину порядка $0.8 \mathrm{~mA} / \mathrm{cm}^{2}$ (AM0) по сравнению со случаем использования AlInP.

Эффект от использования широкозонного слоя GaInP в туннельном диоде, а также результаты оптимизации толщины широкозонного окна для среднего субэлемента были проверены экспериментально. Измеренные спектральные зависимости внешнего квантового выхода фотоответа $\mathrm{Ga}(\mathrm{In}) \mathrm{As}$-субэлементов представлены на рис. 3 . Замена в ТД слоя $n^{++}$-GaAs на слой $n^{++}$-GaInP приводит к возрастанию фоточувствительности во всем спектральном диапазоне (рис. 3, линия 1), а использование не оптимальной толщины широкозонного окна GaInP $(30 \mathrm{~nm})$ заметно понижает уровень фотоответа в диапазоне $700-900 \mathrm{~nm}$ (рис. 3, линии 2 и 3).

В результате проведенных исследований показано, что фотогенерированный ток $\mathrm{Ga}(\mathrm{In})$ As-субэлемента может быть увеличен с начальных 17.8 до $18.2 \mathrm{~mA} / \mathrm{cm}^{2}$ при изменении толщины широкозонного GaInP-окна для среднего субэлемента с 30 до $100 \mathrm{~nm}$ с последующим повышением до $18.9 \mathrm{~mA} / \mathrm{cm}^{2}$ (АМ0) при замене слоя ТД c GaAs на GaInP. Таким образом, комплексные оптимизационные процедуры продемонстрировали потенциал для роста фототока более чем на $6 \%$. При этом такая оптимизация дает потенциал для увеличения КПД многопереходных СЭ на 1.5 абсолютных процента.

\section{Список литературы}

[1] King R.R., Law D.C., Fetzer C.M., Sherif R.A., Edmondson K.M., Kurtz S., Kinsey G.S., Cotal H.L., Krut D.D., Ermer J.H., Karam N.H. // Proc. of the 20th EPVSEC. Barcelona, Spain, 2005. P. 118-123.

[2] King R.R., Law D.C., Edmondson K.M., Fetzer C.M., Kinsey G.S., Yoon H., Sherif R.A., Karam N.H. // Appl. Phys. Lett. 2007. V. 90. P. 183516.

[3] Guter W., Schone J., Philipps S.P., Steiner M., Siefer G., Wekkeli A., Welser E., Oliva E., Bett A.W., Dimroth F. // Appl. Phys. Lett. 2009. V. 94. P. 223504.

[4] Barrutia L., García I., Barrigón E., Ochoa M., Lombardero I., Hinojosa M., Caño P., Bautista J., Cifuentes L., ReyStolle I., Algora C. // Proc. of the 2018 Spanish Conf. on electron devices (CDE). IEEE, 2018. P. 8596996 (1-4). https://doi.org/10.1109/CDE.2018.8596996

[5] Theristis M., O'Donovan T.S. // Solar Energy. 2015. V. 118. P. 533-546.

[6] Heini M., Aierken A., Li Z.H., Zhao X.F., Sailai M., Shen X.B., Xu Y., Liu H.T., Li Y.D., Guo Q., Liu C.M. // AIP Adv. 2018. V. 8. P. 105022.

[7] Kim J.-H., Choi E.Y., Kim B.-J., Han E., Park N. // Vacuum. 2019. V. 162. P. 47-53.

[8] Green M.A., Emery K., Hishikawa Y., Warta W., Dunlop E.D. // Prog. Photovolt.: Res. Appl. 2015. V. 23. P. 1-9.

[9] Stan M.A., Sharps P.R., Fatemi N.S., Spadafora F.S., Aiken D.J., Hou H.Q. // Proc. 28th IEEE PV Specialists Conf. IEEE, 2000. P. 1374-1377.

[10] Минтаиров С.А., Андреев В.М., Емельянов В.М., Калюжный Н.А., Тимошина Н.К., Швари, М.З., Лантратов В.М. // ФТП. 2010. Т. 44. В. 10. С. 1118-1123.

[11] Kalyuzhnyy N.A., Evstropov V.V., Lantratov V.M., Mintairov S.A., Mintairov M.A., Gudovskikh A.S., Luque A., Andreev V.M. // Int. J. Photoenergy. 2014. V. 2014. P. 836284.

[12] Abelés F. // Ann. de Phys. 1950. V. 12. P. 596-640.

[13] Васильев А.М., Ландсман А.П. Полупроводниковые преобразователи. М.: Сов. радио, 1971. 248 с.

[14] Andreev V.M., Emelyanov V.M., Kalyuzhnyy N.A., Lantratov V.M., Mintairov S.A., Shvarts M.Z., Timoshina N.K. // Proc. 23th EPVSEC. Valencia, Spain, 2008. P. 375-381.

\section{Конфликт интересов}

Авторы заявляют, что у них нет конфликта интересов. 